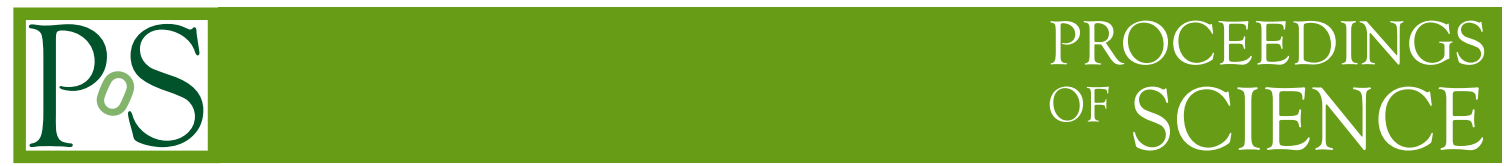

\title{
Recent Results from the HiRes Experiment
}

\section{Andreas Zech* for the HiRes Collaboration}

LPNHE, France

E-mail:aszech@lpnhep.in2p3.fr

The High Resolution Fly's Eye (HiRes) collaboration has measured the spectrum of ultra-high energy cosmic rays (UHECR) in the energy range from $10^{17.2}$ to $10^{20.2} \mathrm{eV}$ with two air fluorescence detectors. Two spectral features have been found: The "ankle" is clearly seen at about $10^{18.5} \mathrm{eV}$. We have also evidence for a suppression of the cosmic ray flux at an energy of about $10^{19.8} \mathrm{eV}$, where the GZK effect is expected to become apparent in the spectrum. The composition of cosmic rays has been measured between $10^{18.0}$ and $10^{19.4} \mathrm{eV}$ using the $\mathrm{X}_{\max }$ method. A measurement of the proton-air inelastic cross section has been made. We have also searched for anisotropy in the arrival directions of ultra-high energy cosmic rays. Our latest results on these topics will be presented.

International Europhysics Conference on High Energy Physics

July 21st-27th 2005

Lisboa, Portugal

${ }^{*}$ Speaker. 


\section{Introduction}

The HiRes experiment is sensitive to the high energy end of the cosmic ray spectrum (above $\sim 10^{17} \mathrm{eV}$ ). In the observed energy range, we expect to see a change from galactic to extragalactic sources, since there are no known mechanisms in our galaxy to provide acceleration to these ultrahigh energies. We also expect to see features from the interactions between charged cosmic ray particles and the photons of the cosmic microwave background (CMB) radiation (photo-spallation; $\mathrm{e}^{+} \mathrm{e}^{-}$pair production; pion photoproduction, known as the "GZK effect"). This paper gives a brief summary of the most recent results from our studies of UHECR with HiRes. The interested reader is referred to more detailed publications.

\section{The HiRes experiment}

The two air fluorescence detectors of the HiRes experiment, located in the desert of Utah, USA, have been described in detail in [1] and [2]. UHECR initiate cascades of secondary charged particles ("air showers") in the atmosphere, which can be observed by the fluorescence light emitted from excited nitrogen molecules in the path of the showers. The shower geometry and the energy of the primary cosmic ray particle are reconstructed from the recorded pulses and times of triggered photomultiplier tubes. Monte Carlo simulations of the air showers and the detectors are used to model the energy dependent aperture. Our reconstruction and simulation methods are explained in [3] and [4].

\section{Monocular spectra}

The updated result of the HiRes measurement of the UHECR energy spectrum in "monocular" mode is shown in Figure 1. The separate analysis of data from the two detectors covers a wider energy range and provides larger statistics at the high energy end than the "stereoscopic" analysis, which selects only events seen in both detectors and thus yields a superior resolution. We have applied a power law fit with two floating break-points to the monocular spectra, resulting in a significantly better $\chi^{2}$ than a fit with a single or no break-points. The positions of the break-points provide the location of the "ankle" (at $10^{18.5} \mathrm{eV}$ ) and of a high energy break in the spectrum. The significance of the observed break at $10^{19.8} \mathrm{eV}$ can be estimated by comparing the prediction of a spectrum that would continue unabated to the highest energies with the fit to the observed spectra [5]. With the updated spectra, we can claim evidence for a high energy break at the $4.8 \sigma$ level.

\section{Composition \& fits to the monocular spectra}

Figure 2 shows the HiRes stereo measurement of the UHECR composition [6]. The mean atmospheric depth $\left(\mathrm{X}_{\max }\right)$ of air showers of a certain energy is used as an indicator for the primary particle species. The comparison of the measured $X_{\max }$ to model predictions indicates a predominantly light composition in the energy range observed by HiRes in stereoscopic mode. We have used this measurement together with the HiRes/MIA result at lower energies [7] in a model we 
fit to the monocular spectra. This model assumes that the shift to a lighter composition observed by HiRes/MIA corresponds to a shift from galactic to extragalactic cosmic rays. The latter are assumed to arrive from uniformly distributed sources and to experience interactions with the CMB and red-shifting. The fit to our data, shown in Figure 3, explains the steepening of the flux below the "ankle" as a result of the $\mathrm{e}^{+} \mathrm{e}^{-}$pair production with the CMB photons and the observed high energy break as a result of the GZK effect.

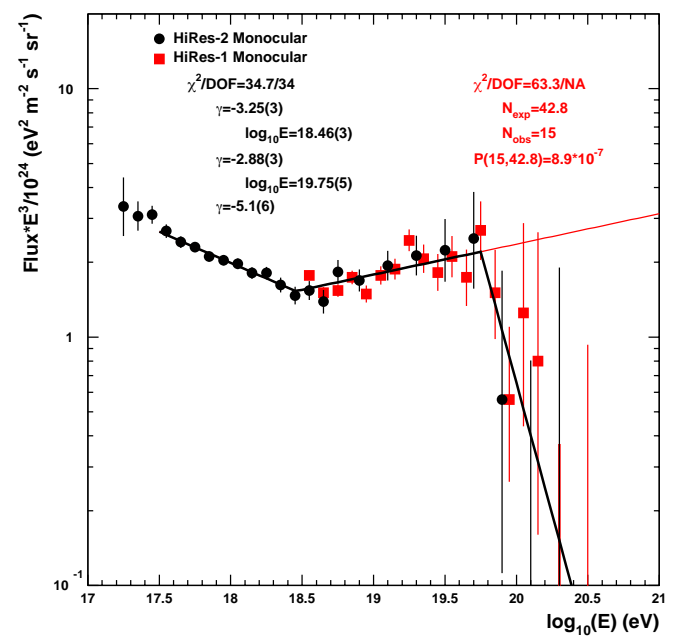

Figure 1: Monocular spectra from HiRes-I (red squares) and HiRes-II (black circles) with broken power law fit.

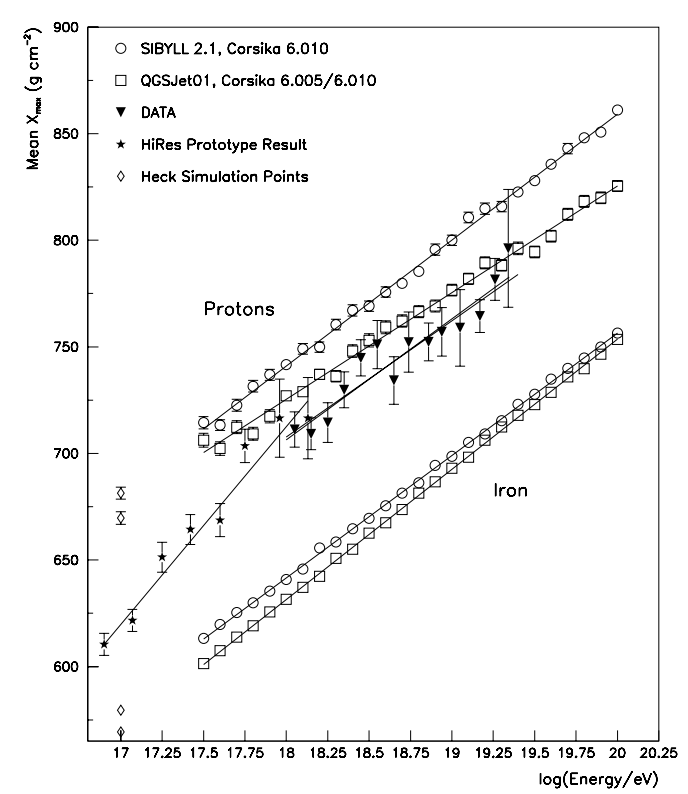

Figure 2: Composition measurement with HiRes Stereo (triangles). The HiRes-MIA measurement is also included (stars). Figure taken from [6].

\section{Measurement of the p-air cross section}

We have measured the inelastic proton-air cross section with HiRes stereo data using a new deconvolution method presented in [8]. The pp cross section that was derived from our measurement can be seen in figure 4 in comparison with results from other cosmic ray experiments and with an extrapolation of the Block-Halzen fit [9] to accelerator data. Our result agrees with the fit.

\section{Correlations with BL Lac objects}

Previous claims of correlations between BL Lacs and cosmic ray arrival directions based on AGASA and Yakutsk data have been excluded in our first study of this subject. However, a new correlation has been observed for HiRes stereo data above $10^{19} \mathrm{eV}$. This a posteriori correlation awaits confirmation with an independent set of HiRes data of comparable statistics, which will be available in 2006. A discussion of the tests of previous claims and the new hypothesis of a potential BL Lac correlation can be found in [10]. 


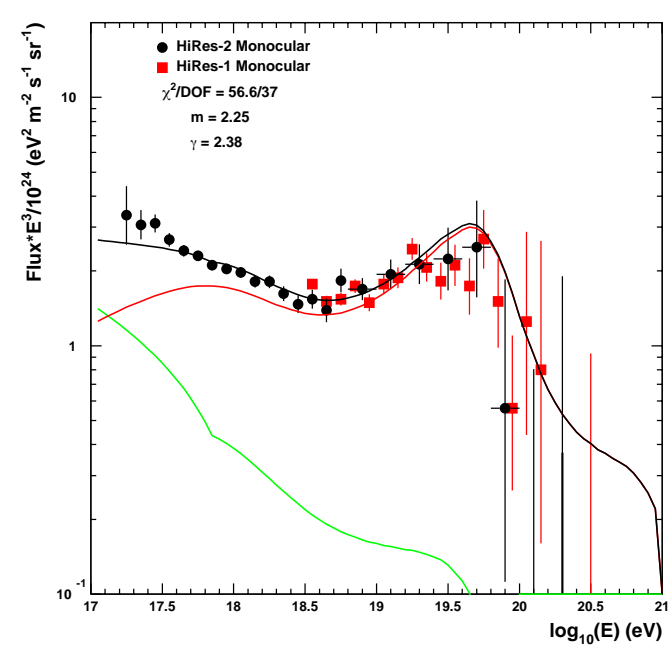

Figure 3: Monocular spectra from HiRes-I (red squares) and HiRes-II (black circles), fitted with a model of a galactic (green line) and an extragalactic (red line) component. More explanations in the text.

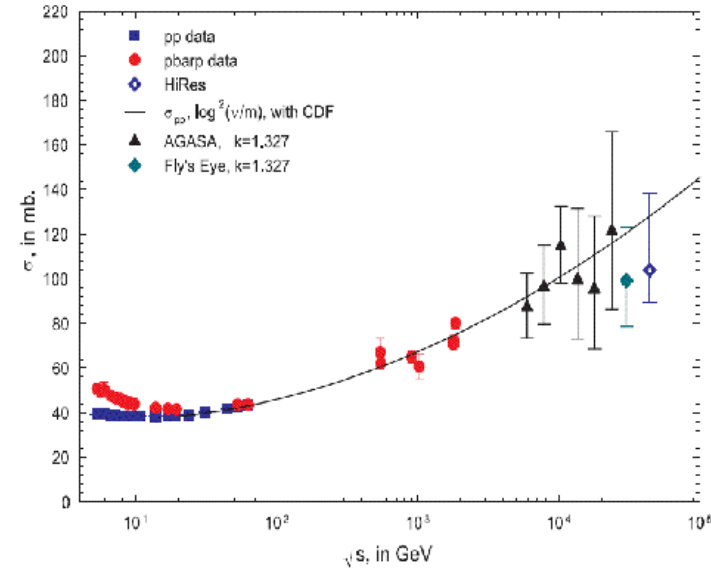

Figure 4: HiRes measurement of the pp cross section (open diamond). The Block-Halzen fit to accelerator data, as well as other cosmic ray measurements are shown in comparison.

\section{Acknowledgements}

This work is supported by US NSF grants PHY-9321949, PHY-9322298, PHY-9904048, PHY9974537, PHY-0098826, PHY-0140688, PHY-0245428, PHY-0305516, PHY-0307098, and by the DOE grant FG03-92ER40732. We gratefully acknowledge the contributions from the technical staffs of our home institutions. The cooperation of Colonels E. Fischer and G. Harter, the US Army, and the Dugway Proving Ground staff is greatly appreciated.

\section{References}

[1] T. Abu-Zayyad et al., Proceedings of the 26th International Cosmic Ray Conference, 5, 349 (1999)

[2] J. Boyer, B. Knapp, E. Mannel and M. Seman, Nucl. Inst. and Meth. A 482, 457 (2002)

[3] R. U. Abbasi et al., Phys. Rev. Lett., 92, 151101 (2004), [astro-ph/0208243]

[4] R. U. Abbasi et al., Astropart. Phys., 23, 157 (2005), [astro-ph/0208301]

[5] R. U. Abbasi et al., Phys. Lett. B 619, 271 (2005), [astro-ph/0501317]

[6] R.U. Abassi et al., Astrophys. J., 622 (2005) 910-926, [astro-ph / 0407622 ]

[7] T. Abu-Zayyad et al., Phys. Rev. Lett., 84, 19, 4276 (2000), [astro-ph/ 9911144 ]

[8] K. Belov for HiRes, Proceedings of the 29th International Cosmic Ray Conference

[9] M.M.Block, F.Halzen, Phys. Rev. D, 63, 11, 114004 (2001)

[10] R.U.Abbasi et al., accepted by Astrophys. J. (2005), astro-ph/ 0507120 